\title{
As a host society to SFRRI 2014 in Kyoto
}

\author{
Osamu Handa, ${ }^{1,2, *}$ Yuji Naito ${ }^{1,3}$ and Shinya Toyokuni ${ }^{3,4}$ \\ 'Department of Molecular Gastroenterology and Hepatology, Kyoto Prefectural University of Medicine, \\ 465 Kajiicho, Kawaramachi-Hirokoji, Kamigyo-ku, Kyoto 602-8566, Japan \\ 2Secretary General of SFRRI 2014 \\ ${ }^{3}$ Chair of SFRRI 2014 \\ ${ }^{4}$ Department of Pathology and Biological Responses, Nagoya University Graduate School of Medicine, 65 Tsurumai-cho, Showa-ku, Nagoya 466-8550, Japan
}

It is our great honor to host the 17th Biennial Meeting of Society for Free Radical Research International (SFRRI 2014) that is being held at the Kyoto International Conference Center, in Kyoto, Japan on March 23rd-26th, 2014. Previously we had this biennial meeting twice in Kyoto, in the year 1988 and 2000. This meeting has a great meaning not only for every member in SFRRI but also for those in SFRR Japan, since the SFRRI 1998 meeting was a key event behind the establishment of SFRR Japan. Since 1998, SFRR Japan has expanded the number of the members and has developed a variety of original research activities.

The International Scientific Program Committee (ISPC) representing each region of SFRR has worked hard all together to develop what we believe will be an exciting and informative meeting to both young and senior participants. As a result, the congress will include award lectures and symposia on novel aspects of free radical research, which was democratically selected after the application during December 2012 and January 2013 by the voting of ISPC. Concurrent symposia will address in-depth and cutting-edge scientific topics in free radical chemistry, biology and medicine. In addition, the meeting will have four plenary lectures presented by Prof. Rafael Radi (Uruguay), Prof. Harold. M Swartz (USA), Prof. Arturo Zychlinsky (Germany) and
Prof. Hideyuki Saya (Japan). Each lecture would reveal novel aspects of free radical research.

It has been our sincere wish to make the meeting affordable and attractive especially to young scientists. There will be several awards from each region of SFRR as well as from this meeting. Sunrise lecture for the beginner of free radical research will be presented. Several satellite meetings are now planned in order to allow participants with special interests to meet.

The congress venue, Kyoto city, is a UNESCO World Heritage site with a long and rich history going back over 1,200 years and was the original imperial capital of Japan. With more than 3,000 temples and shrines, full of traditional Japanese culture, with cherry blossoms presumably in full bloom, Kyoto city will deeply inspire your sixth sense. Besides traditional atmosphere, Kyoto city is thriving and exciting modern city with excellent facilities and easy links to the rest of the world. We believe that SFRRI 2014 meeting will be rich in science, and a great opportunity to hear cutting edge advances in the free radical research, as well as an opportunity to renew old friendships and establish new collaborations and associations.

We look forward to meeting all of you in Kyoto in March 2014. 